\title{
Late-onset peripheral joint disease in ankylosing spondylitis
}

\author{
MARCD. COHEN AND WILLIAM W. GINSBURG \\ From the Division of Rheumatology and Internal Medicine, Mayo Clinic and Mayo Foundation, Rochester, \\ Minnesota, USA
}

SUMMARY We reviewed the records of 150 patients with definite ankylosing spondylitis who had significant peripheral arthritis and were seronegative and found 7 patients who had the onset of peripheral arthritis after their spinal disease became inactive. This late-onset peripheral arthritis may lead to significant joint deformity, and aggressive therapy may be warranted.

Ankylosing spondylitis is a chronic inflammatory disease that predominantly affects the axial skeleton. Axial arthritis with invariable sacroiliac joint involvement, an association with the histocompatibility antigen HLA B27, and a male preponderance further characterise the disease. In addition many patients with ankylosing spondylitis have peripheral joint involvement at some stage of the disease. Inflammatory changes in the peripheral joints manifested by swelling, tenderness, and limitation of movement have been estimated to occur in between 30 and $50 \%$ of patients, ${ }^{12}$ and if the shoulders and hips are included the incidence may be higher than $60 \% .^{34}$ The large joints are believed to be the most frequently affected, with the order in decreasing frequency reported as hips, shoulders, knees, wrists, and metacarpophalangeal (MCP), metatarsophalangeal (MTP), and rarely proximal interphalangeal (PIP) joints. ${ }^{5}$ Although peripheral joint involvement, particularly of the hips or knees, may antedate back pain in about $20 \%$ of patients, ${ }^{5}$ peripheral joint symptoms more frequently occur simultaneously or shortly after the onset of axial arthritis.

Recently we saw a patient in whom significant peripheral joint disease consistent with ankylosing spondylitis developed approximately 30 years after the axial symptoms had resolved. We reviewed the records of 150 patients with definite ankylosing spondylitis who had significant peripheral arthritis and were seronegative and found 7 patients who had the onset of peripheral arthritis after their spinal disease became inactive.

Accepted for publication 3 November 1981.

Correspondence to Dr Marc D. Cohen, c/o Section of Publications, Mayo Clinic, 200 First Street SW, Rochester, MN 55905, USA.

\section{Case reports}

CASE 1

A 67-year-old white man had back pain since the age of 24 years. He had persistent pain and stiffness until the age of 31 years, when he was told that he had rheumatoid spondylitis and was given $x$-ray treatment to his back. Despite severe limitation of motion of the spinal column he did well until the age of 60 years, when he suffered pain and swelling of the knees, ankles, feet, wrists, and hands. He was seen at our clinic several years later with progressive symptoms. Physical examination revealed dorsal kyphosis of the spinal column, with essentially no movement. The only motion at the hips was $10^{\circ}$ of flexion. He had synovitis at the knees, ankles, and wrists, and these joints had severely limited motion. He had synovitis and early subluxation at several MCP, PIP, and MTP joints (Fig. 1, upper). Laboratory examination revealed a haemoglobin level of 10 $\mathrm{g} / \mathrm{dl}$, an erythrocyte sedimentation rate of $60 \mathrm{~mm}$ in 1 hour, and persistently negative tests for antinuclear antibodies and rheumatoid factor. The patient was positive for HLA B27 antigen. Roentgenograms showed bony fusion of the posterior elements along the entire spinal column, with extensive calcification of the anterior longitudinal ligament. The sacroiliac joints were completely ankylosed. Other views demonstrated extensive erosive changes at the knees, wrists, ischial tuberosities, and MCP joints (Fig. 1, lower). Symmetrical loss of joint space and diffuse osteoporosis were also present.

CASE 2

A 75-year-old man had had mild sacroiliac pain since 


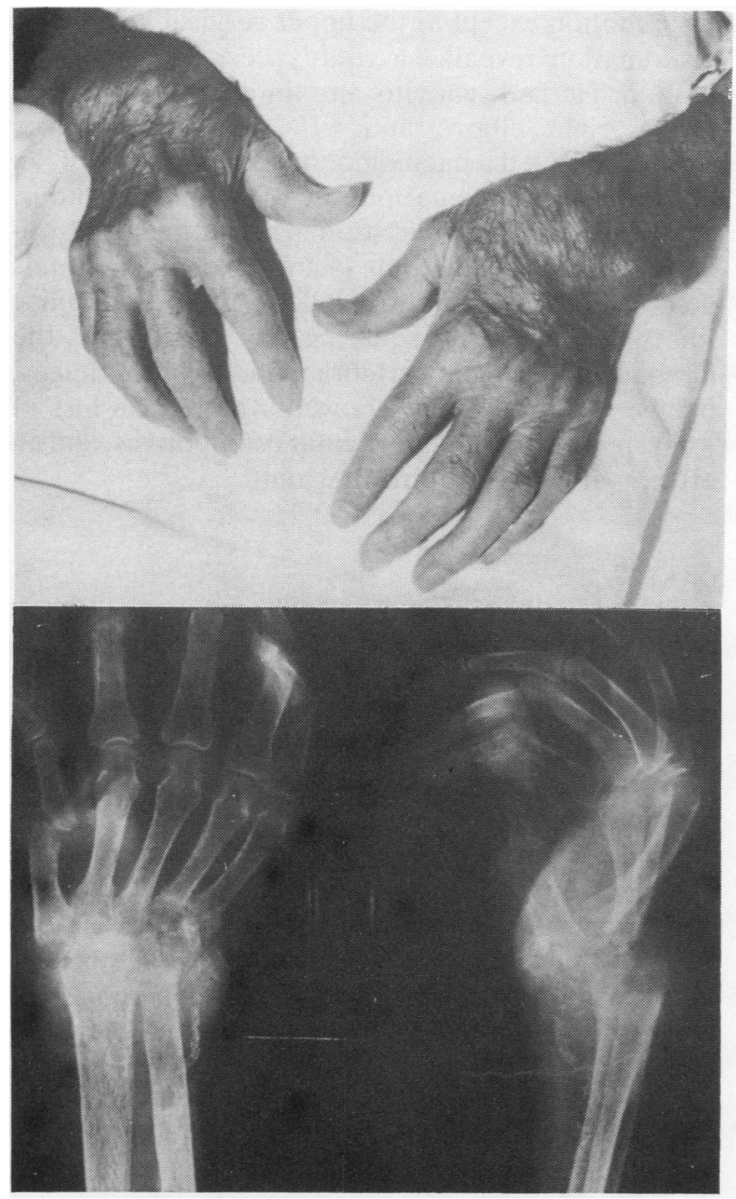

Fig. 1 Upper: Swelling of wrists and metacarpophalangeal joints. Lower: Severe destruction and deformity. Erosions at several metacarpophalangeal joints.

the age of 20 years. By the age of 30 years he had pain in almost his entire thoracic and lumbar spinal column and also had pronounced stiffness. By the age of 35 years his spinal movement was limited, but he experienced no further pain. He did well until the age of 72 years, when he presented to his local physician with left hip pain, bilateral shoulder pain, and left ankle pain. Low-dose corticosteroid therapy was eventually initiated. He was seen at our clinic at the age of 75 years, with symptoms that had progressively worsened. Physical examination revealed dorsal kyphosis of the spinal column, with essentially no motion except for a minimal degree at the upper cervical joints. He had synovitis in both shoulders and his left knee and ankle. The left hip was painful and was severely limited in range of motion. Laboratory studies revealed a haemoglobin level of $12 \cdot 1 \mathrm{~g} / \mathrm{dl}$, an erythrocyte sedimentation rate of $67 \mathrm{~mm}$ in 1 hour, a negative test for antinuclear antibody, and a negative test for rheumatoid factor. Roentgenograms of the pelvis demonstrated obliteration of the sacroiliac joints, and views of the spinal column demonstrated advanced changes, typical of ankylosing spondylitis, with squaring of vertebral bodies, bridging syndesmophytes, and calcification of the anterior longitudinal ligament. Roentgenograms of both shoulders, knees, and hips showed erosive changes with periarticular osteoporosis and joint space narrowing. The patient subsequently underwent a left total hip arthroplasty, and histological examination of the synovial membrane disclosed chronic inflammatory synovitis.

\section{CASE 3}

A 54-year-old woman first experienced discomfort, stiffness, and limitation of motion of the lower back at the age of 19 years. She suffered for 5 years, but by the age of 24 years the pain had resolved. She did well until the age of 32 years, when she presented at our clinic with pain in her right knee, temporomandibular joints, cervical spinal column, and shoulders. She had approximately 1 hour of morning stiffness. She has been followed up for more than 20 years and subsequently developed additional pain and swelling in her knees, ankles, and wrists and MCP, PIP, and MTP joints. Physical examinations at various times revealed limitation of motion of the lumbar, thoracic, and cervical spinal column. She had synovitis in her knees, ankles, and wrists and MCP and PIP joints and had tenosynovitis of the flexor tendons of her left hand. Laboratory studies revealed a haemoglobin level of between 10 and $12 \mathrm{~g} / \mathrm{dl}$, an erythrocyte sedimentation rate of between 60 and $90 \mathrm{~mm}$ in 1 hour, an elevated $\gamma$-globulin level in the range of 2.5 to $3.10 \mathrm{~g} / \mathrm{dl}$, and repeatedly negative tests for antinuclear antibodies and rheumatoid factor. She was positive for HLA B27 antigen. Roentgenograms revealed solid bony ankylosis of the sacroiliac joints, and views of the spinal column demonstrated advanced changes of ankylosing spondylitis. Views of her knees, temporomandibular joints, hands, and feet showed small erosions, loss of joint space, and juxta-articular osteoporosis. After right total knee arthroplasty, histological examination of the synovial membrane revealed changes consistent with chronic inflammatory synovitis.

CASE 4 .

An 83-year-old white woman initially had low-back pain at the age of 26 years and shortly afterward had pain and swelling of her knees. During the next 2 years she experienced progressively less mobility in her back and painful limitation of motion at the knees. By the age of 30 years she had no further pain 
or swelling but had residual restriction of motion of her knees and spinal column. She did well until the age of 61 years, when she developed painful swelling of her knees and ankles, which was controlled with rest and aspirin. At the age of 81 years she presented at our clinic with complaints of increasing morning stiffness and painful swelling of her shoulders, wrists, fingers, ankles, and feet. Physical examination revealed severely limited spinal movement. She had flexion contractures at both knees and both hips and had synovitis at the ankles, shoulders, and wrists and MCP and PIP joints. Laboratory studies revealed a haemoglobin level of $12.1 \mathrm{~g} / \mathrm{dl}$, an erythrocyte sedimentation rate of $66 \mathrm{~mm}$ in 1 hour, negative tests for lupus erythematosus cells, and multiple negative tests for rheumatoid factor. An electromyelogram revealed findings consistent with bilateral carpal tunnel syndromes. Roentgenograms showed complete ankylosis of the sacroiliac joints. Views of the spinal column revealed syndesmophytes with squaring and bridging of the thoracic and lumbar vertebrae. Other roentgenograms showed erosive changes at the hips and wrists, with periarticular deossification and symmetrical narrowing of the joint spaces there and at the shoulders and MCP and PIP joints.

\section{CASE 5}

A 62-year-old white man developed mild low-back pain at the age of 24 years and experienced mild, self-limited recurrences approximately every 4 to 5 years. At the age of 51 years he experienced severe low-back pain and began taking aspirin for relief. At the age of 61 years he again experienced low-back pain accompanied by painful swelling of his knees, wrists, and fingers. Physical examination revealed only mildly decreased range of motion at the spinal column. There was active synovitis at the knees and wrists and MCP and PIP joints. Laboratory studies revealed a haemoglobin level of $13.5 \mathrm{~g} / \mathrm{dl}$, an erythrocyte sedimentation rate of $35 \mathrm{~mm}$ in 1 hour, negative tests for antinuclear antibodies, and negative tests for rheumatoid factor. The patient was positive for HLA B27 antigen. Roentgenograms revealed ankylosis of the sacroiliac joints and squaring of the lumbosacral vertebrae. Views of the hands and wrists showed periarticular osteoporosis, symmetrical joint space narrowing, and marginal erosions.

\section{CASE 6}

A 53-year-old white man developed back pain at the age of 27 years, but after several years the pain resolved. He did well until the age of 52 years, when pain developed in his temporomandibular joints, hips, knees, shoulders, hands, and right elbow. Physical examination revealed a fixed spinal column with little motion except at the upper cervical levels. Hip examination revealed a greatly decreased range of motion. He had synovitis and limited motion at the knees, right elbow, and PIP joints. Laboratory studies revealed a haemoglobin level of $13.3 \mathrm{~g} / \mathrm{dl}$, an erythrocyte sedimentation rate of $50 \mathrm{~mm}$ in 1 hour, and repeatedly negative studies for antinuclear antibodies and rheumatoid factor. Roentgenograms demonstrated complete ankylosis of the sacroiliac joints and advanced squaring and bridging of the lumbar and thoracic vertebrae. Views of the knees, hands, and right elbow showed symmetrical loss of joint space, early juxta-articular osteoporosis, and an erosion at the right third PIP joint.

\section{CASE 7}

A 51-year-old white man began having low back and right hip pain at the age of 17 years, and by the age of 25 years he also had pain and swelling in his temporomandibular joints, shoulders, elbows, hands, hips, knees, and ankles. He was told he had rheumatoid spondylitis and was given $x$-ray treatment to his back and hip region. His symptoms were mostly controlled by aspirin until he experienced increased hip pain at the age of 35 years, when he underwent bilateral total hip arthroplasties. He did well until the age of 43 years, when he noted persistent swelling in his right knee and underwent total synovectomy. At the age of 45 years pain and swelling developed in his ankles, left shoulder, and elbows and MCP and PIP joints, and he was seen at our clinic. Physical examination revealed an essentially immobile spinal column. He had severe limitation of motion at his hips and knees. Synovitis was present at the shoulders, elbows, and ankles and MCP, PIP, and MTP joints. Laboratory examination revealed a haemoglobin level of $11.6 \mathrm{~g} / \mathrm{dl}$, an erythrocyte sedimentation rate of $54 \mathrm{~mm}$ in 1 hour, and several negative results for antinuclear antibodies and rheumatoid factor. Roentgenograms revealed complete ankylosis of the sacroiliac joints, with advanced changes of ankylosing spondylitis along most of the spinal column. Views of the shoulders, elbows, hands, and feet showed joint space narrowing, periarticular osteoporosis, and several marginal erosions.

\section{Discussion}

The patients described fulfilled the criteria for definite ankylosing spondylitis. $^{6}$ All had severe peripheral joint disease, some with significant residual disability. That these patients had extensive involvement of their peripheral joints is not unexpected, but the lateness of their peripheral joint manifestations in relation to their axial disease is 
unusual and noteworthy, and created some reservations about the interpretation that their peripheral arthritis was secondary to ankylosing spondylitis.

Ankylosing spondylitis and rheumatoid arthritis have several features in common. One disease can manifest clinical features more commonly associated with the other. Probably more than a third of the patients with ankylosing spondylitis have peripheral involvement at some stage of their disease, and this can be clinically identical to that seen in rheumatoid arthritis. ${ }^{7}$ The synovitis of ankylosing spondylitis is also histologically identical to that of rheumatoid arthritis. ${ }^{1}$ Cellular infiltration of the synovial membrane, synovial villous proliferation, and eventual pannus formation occur in both diseases, leading to cartilage destruction and joint space narrowing. This involvement is sometimes not progressive and may resolve completely, but it can result in permanent damage.

This basic similarity between the underlying pathological changes in the peripheral joints in ankylosing spondylitis and rheumatoid arthritis helps to explain the overlap in clinical and radiographic features. Demineralisation, joint space narrowing, and erosive changes can be seen in both disorders. In a study of 25 patients with ankylosing spondylitis and peripheral joint disease Resnick $^{2}$ noted several differences in the radiographic appearance of the 2 diseases. Ankylosing spondylitis was more likely to produce unilateral or asymmetrical joint involvement, bony ankylosis without adjacent erosions, subchondral sclerosis, and periosteal inflammation. In contrast rheumatoid arthritis was more likely to produce symmetrical joint space involvement, demineralisation, larger erosions and subchondral cysts, and subluxations. Somewhat more common in ankylosing spondylitis than in rheumatoid arthritis were abnormalities of the distal interphalangeal joints in combination with other finger joints, erosions at the interphalangeal joints of the great toe and the first tarsometatarsal joint, and shoulder alterations. Although helpful as guidelines, these distinctions may be subtle, and overlap between the peripheral $x$-ray appearances of these disorders exists.

Besides sharing common features there are at least 18 reported cases of definite rheumatoid arthritis coexisting with ankylosing spondylitis. ${ }^{89}$ As Clayman and Reinertsen ${ }^{8}$ emphasised, many of the reports involved patients who had ankylosing spondylitis as young men and who later, when their spondylitis was inactive, developed rheumatoid arthritis. None of the previously published case reports included patients whose spondylitis worsened after the onset of rheumatoid arthritis. When the 2 disorders coexist, they do not seem to interact. ${ }^{8}$ This pattern of lateonset peripheral joint disease in patients with mostly inactive spondylitis is similar to that of several of the patients we describe. The main difference is that the previously reported patients were seropositive, and many also had rheumatoid nodules.

When conservative rates for the prevalence of rheumatoid arthritis and ankylosing spondylitis were used, Luthra et al. ${ }^{10}$ predicted that the 2 diseases could coexist in 1 per 238000 persons. Using sexspecific rates for concurrent disease in adults having one or the other disorder Clayman and Reinertsen ${ }^{8}$ predicted that approximately 1 in 100 patients with ankylosing spondylitis will have coexisting rheumatoid arthritis. Both of these estimates suggest that the coexistence is more common than is at present appreciated and reported. This discrepancy may be due to some negative influence in the expression of one disorder by the other. ${ }^{8}$ It also may be due to the fact that milder or less classic forms of the coexistent disease are not detected.

The 7 patients in our series had ankylosing spondylitis with significant late-onset seronegative peripheral joint disease. Examination of the affected peripheral joints revealed active synovitis. None of the patients was positive for rheumatoid factor, and none had rheumatoid nodules. Roentgenograms revealed juxta-articular osteoporosis, joint space narrowing, and small erosions. All were treated conservatively, most with anti-inflammatory medication

Table 1 Characteristics of patients with late-onset peripheral joint disease in ankylosing spondylitis

\begin{tabular}{|c|c|c|c|c|c|c|c|}
\hline Case & $\begin{array}{l}\text { Age at onset of } \\
\text { spondylitis (yr) }\end{array}$ & $\begin{array}{l}\text { Age at onset of } \\
\text { peripheral arthritis (yr) }\end{array}$ & Sex & $\begin{array}{l}X \text {-ray evidence of } \\
\text { spondylitis }\end{array}$ & $\begin{array}{l}+v e \text { for } \\
H L A B 27\end{array}$ & $\begin{array}{l}\text { Rheumatoid } \\
\text { factor }\end{array}$ & $\begin{array}{l}\text { Location of peripheral } \\
\text { arthritis* }^{*}\end{array}$ \\
\hline 1 & 24 & 60 & $\mathbf{M}$ & Yes & Yes & No & MCPs, wrists, knees, ankles \\
\hline 2 & 20 & 72 & $\mathbf{M}$ & Yes & Not done & No & Shoulders, hips, knees, ankles \\
\hline 3 & 19 & 32 & $\mathbf{F}$ & Yes & Yes & No & $\begin{array}{l}\text { TMJ, PIPs, MCPs, wrists, knees } \\
\text { ankles, feet }\end{array}$ \\
\hline 4 & 26 & $26 / 61+$ & $\mathbf{F}$ & Yes & Not done & No & $\begin{array}{l}\text { PIPs, MCPs, wrists, shoulders } \\
\text { hips, ankles }\end{array}$ \\
\hline 5 & 24 & 61 & $\mathbf{M}$ & Yes & Yes & No & PIPs, MCPs, wrists knees \\
\hline 6 & 27 & 52 & $\mathbf{M}$ & Yes & Not done & No & PIPs, right elbow, knees \\
\hline 7 & 17 & $25 / 45+$ & $\mathbf{M}$ & Yes & Not done & No & $\begin{array}{l}\text { PIPs, MCPs, elbows, shoulders, } \\
\text { ankles, MTPs }\end{array}$ \\
\hline
\end{tabular}

*MCP=metacarpophalangeal; $T M J=$ temperomandibular; $P I P=$ proximal interphalangeal; $M T P=$ metatarsophalangeal.

tTwo distinct episodes of peripheral arthritis: the first clearly associated with the onset of spondylitis, the second significantly later. 
and physiotherapy. Several have residual deformities and restricted motion of peripheral joints.

Late-onset seronegative peripheral arthritis associated with ankylosing spondylitis is unusual. Of the 150 patients with definite ankylosing spondylitis who had peripheral arthritis and were seronegative whose records we reviewed only $4 \cdot 7 \%$ ( 7 patients) had the peripheral arthritis manifest after the axial joints had ankylosed and the axial symptoms abated (Table 1). Nevertheless, late-onset seronegative peripheral arthritis can be significant in patients with ankylosing spondylitis and remains a vexing problem as to its classification. Whether it represents a separate, coexistent disease and should be labelled seronegative rheumatoid arthritis or represents the peripheral arthritis of ankylosing spondylitis is unclear. The findings in cases 4 and 7 suggest that it is part of the spectrum of ankylosing spondylitis, because both patients had peripheral arthritis at the time of the initial involvement of the spinal column, which then became quiescent, only to become active many years later after the spinal disease was inactive.

There may be therapeutic implications in distinguishing between the peripheral arthritis of ankylosing spondylitis and a coexistent seronegative rheumatoid arthritis. With significant synovitis unresponsive to anti-inflammatory medications, the use of gold or penicillamine would be considered in a patient with seronegative rheumatoid arthritis. Neither of these agents is considered useful in ankylosing spondylitis, but usually this relates to the spinal disease. There have been no studies on the use of gold or penicillamine for the peripheral arthritis of ankylosing spondylitis, but in patients with significant peripheral synovitis, especially if erosions are present on roentgenograms, a course of gold or penicillamine probably is warranted.

Besides the above-reported 7 cases we have seen the onset of a peripheral inflammatory arthritis in patients more than 40 years old who are negative for rheumatoid factor. In many instances no underlying disease was found, such as psoriasis, inflammatory bowel disease, or systemic lupus erythematosus. However, we are impressed that some of these patients will have bilateral sacroiliitis without spinal involvement and are positive for HLA B27. Frequently, their back symptoms are minimal and had usually occurred many years previously. The findings in these cases suggest that late-onset peripheral arthritis associated with ankylosing spondylitis or sacroiliitis alone may be more frequent than previously recognized.

\section{References}

1 Cruickshank B. Pathology of ankylosing spondylitis. Clin Orthop 1971; 74: 43-58.

${ }^{2}$ Resnick D. Patterns of peripheral joint disease in ankylosing spondylitis. Radiology 1974; 110: 523-32.

${ }^{3}$ McEwen C, DiTata D, Lingg C, Porini A, Good A, Rankin T. Ankylosing spondylitis and spondylitis accompanying ulcerative colitis, regional enteritis, psoriasis and Reiter's disease: a comparative study. Arthritis Rheum 1971; 14: 291-318.

4 Engleman E G, Engleman E P. Ankylosing spondylitis: recent advances in diagnosis and treatment. Med Clin North $A M$ 1977; 61 No. 2: 347-64.

5 Ogryzlo M A, Rosen P S. Ankylosing (Marie-Strümpell) spondylitis. Postgrad Med 1969; 45: 182-8.

${ }^{6}$ Bennett P H, Wood P H N, eds. Population studies of the rheumatic diseases. Section 14: Recommendations. Excerpta Medica International Congress Series No. 148, 1968, pp 456-7.

7 Ogryzlo M A. Ankylosing spondylitis. In: Hollander J L, McCarty D J Jr, eds. Arthritis and Allied Conditions: A Textbook of Rheumatology. 8th ed. Philadelphia: Lea and Febiger, 1972: 699-723.

- Clayman M D, Reinertsen J L. Ankylosing spondylitis with subsequent development of rheumatoid arthritis, Sjögren's syndrome, and rheumatoid vasculitis. Arthritis Rheum 1978; 21: 383-8.

9 Good A E, Hyla J F, Rapp R. Ankylosing spondylitis with rheumatoid arthritis and subcutaneous nodules (letter to the editor). Arthritis Rheum 1977; 20: 1434-7.

10 Luthra H S, Ferguson R H, Conn D L. Coexistence of ankylosing spondylitis and rheumatoid arthritis. Arthritis Rheum 1976; 19: 111-4. 